\title{
Visión Criminológica del delito online grooming.
}

\author{
Criminological view of Online Grooming
}

Alberto Bragado Sobrino

Universidad Rey Juan Carlos, Madrid, España

Email de correspondencia: alberto.bs.criminologia@gmail.com

\section{Resumen}

La literatura cientifica identifica tres tipologías de individuos que perpetran el online grooming: hipersexualizados, adaptativos e intimos. El presente estudio tiene como objetivo explorar el perfil del potencial delincuente que perpetra este delito y sus diferentes tipologías tanto en Estados Unidos como en España. Para ello, se comparó una muestra conjunta de 258 casos de grooming: por un lado, 243 son casos de online grooming en Estados Unidos y, por otro lado, 15 son casos de online grooming en España. Los resultados indican que el online grooming es perpetrado, mayoritariamente, por un hombre de entre veintiuno y treinta años de edad, motivado sexual o afectivamente. El efecto de la prolongación del tiempo entre el contacto y la proposición sexual en relación con las estrategias empleadas por los agresores, refleja los tipos diferenciados de groomers que se encuentran en este fenómeno: intimos, adaptativos e hipersexualizados.

\section{Palabras Clave}

Online grooming, nuevas tecnologías, perfil criminal, estrategias de grooming y criminología

\begin{abstract}
The scientific literature identifies three types of individuals who perpetrate online grooming: hypersexualized, adaptive and intimate. The present study aims to explore the profile of the potential offender who perpetrates this crime and their different typologies both in the United States and Spain. To this end, a joint sample of 258 grooming cases was compared. On the one hand, 243 are cases of online grooming in the United States. On the other hand, 15 are cases of online grooming in Spain. The results indicate that online grooming is mainly perpetrated by a man between twenty-one and thirty years old, sexually or emotionally motivated. The effect of the prolongation of the time between contact and the sexual proposition in relation to the strategies used by the aggressors reflects the different types of groomers found in this phenomenon: intimate, adaptive and hypersexualized.
\end{abstract}

\section{Keywords}

Online grooming, new technologies, criminal profile, grooming strategies and criminology 


\section{INTRODUCCIÓN}

La red consolida un escenario donde confluyen múltiples conductas de explotación sexual a niñas y niños (Maldonado, 2019). Una de las tipologías delictivas en pleno auge y con una tasa de esclarecimiento muy baja debido a las características inherentes a la red es el fenómeno online grooming (Viader, 2020).

Desde una perspectiva criminológica, este fenómeno delictivo es muy complejo de analizar, no solo por el solapamiento que presenta en relación con el amplio abanico de conductas que engloba el Abuso Sexual Infantil (ASI) mediante las Tecnologías de la Información y Comunicación (TICs), sino por la incoherencia de la realidad del online grooming entre la comunidad científica, la Doctrina y la jurisprudencia. Villacampa (2014) realiza una recopilación de la extensa variedad de definiciones existentes en torno a este fenómeno delictivo y las agrupa en tres categorías: (i) aquellas que se centran en la seducción, (ii) las que lo definen como una idea de pedofilia y (iii) las que lo identifican como un proceso para ganar la confianza del menor. La mayoría de la comunidad científica y Doctrina española se posicionan en la tercera agrupación, indicando como elemento clave del online grooming el desarrollo que se produce entre agresor y víctima con la finalidad de obtener la confianza de ésta última (Craven et al., 2006 y Villacampa, 2014).

Este fenómeno se compone principalmente de dos individuos: groomer (denominación del agresor de este delito concreto) y víctima. Estos sujetos interactúan mediante las TICs con la finalidad de conformar una relación de confianza única y exclusiva, para ello el groomer utilizará alguna estrategia tales como la muestra de afecto o cariño, el engaño, la manipulación, o bien, la agresividad o violencia; finalmente, se producirá el contacto sexual, ya sea mediante medio físico o virtual.

La literatura especializada ha definido a los sujetos que perpetran online grooming como un grupo muy variado (Bergen et al., 2014), en oposición a lo que se estimaba, es decir, un individuo "violento, compulsivo, reincidente y pedófilo” (De Santisteban y Gámez-Gaudix, 2017a, p.88). Sin embargo, la seguridad empleada por parte de los groomers como medida de precaución, resalta un conocimiento de ilegalidad en sus acciones (Kloess et al., 2017). Por un lado, el complejo fenómeno del online grooming es un proceso arduo y lento, incluso, puede que el contacto sexual nunca llegue a producirse, por lo que resulta incompatible con agresores impulsivos (De Santisteban y Gámez-Gaudix, 2017a), Briggs et al. (2011) demostraron que los delincuentes sexuales online tenían problemas de compulsividad sexual. Por otro lado, las víctimas de online grooming suelen ser adolescentes, por tanto, la pedofilia tampoco es una característica esencial en esta tipología delictiva (Seto et al., 2012 y De Santisteban y Gámez-Gaudix, 2017a).

En cuanto a la edad de los groomers, se ha observado que los individuos que perpetran este delito cada vez son más jóvenes. Young (2005) establecía la edad media en los treinta y ocho años, sin embargo, otros estudios, más recientes, demuestran que los groomers con menos de veinticinco años de edad son el grupo más numeroso (Villacampa y Gómez, 2016 y De Santisteban y GámezGaudix, 2017b).

Por otro lado, un aspecto importante del perfil es la variable sexo. Este delito se perpetra predominantemente por hombres. Así, Villacampa y Gómez (2016) destacan que en el online grooming cometido por un adulto, el 70\% de los casos lo perpetran hombres y entre iguales, tres de cada cuatro. No obstante, en nuevas investigaciones se aprecia un $25 \%$ de mujeres que cometen esta tipología delictiva (Gámez-Gaudix et al., 2017).

Finalmente, numerosos han sido los estudios que han intentado analizar qué elementos de una víctima captan la atención del groomer (O'Connell, 2003; Tener et al., 2015 y Kloess et al., 2017). Con ese análisis se ha producido una serie de categorizaciones, clasificando, así, a los groomers en uno u otro subgrupo, según la motivación principal del agresor. Webster et al. (2012) identifican tres tipos: (i) aquellos que buscan establecer una auténtica relación de amor, cuidado y afecto hacia el menor, es decir, los íntimos. "Este sujeto no oculta su identidad, puesto que busca una relación sentimental con la víctima y pretende que ésta se enamore de cómo es él realmente" (Maldonado, 2019, p.8); (ii) aquel sujeto que consigue modificar su conducta y adaptarse a la de su víctima, o sea, los adaptativos. "Consideran que el menor es maduro y está capacitado para mantener relaciones sexuales con un adulto. Los encuentros que proponen no tienen como fin una relación sentimental, sino un contacto sexual" (Maldonado, 2019, pp.8-9); (iii) aquellos que no buscan un acercamiento al menor, sino encuentros sexuales inmediatos. "Sujeto cuyo único propósito sería tener encuentros inmediatos sin invertir mucha dedicación y tiempo" (De Santisteban y Gámez-Gaudix, 2017a, p.91).

Respecto a la edad de las víctimas, Montiel et al. (2014) muestran en su estudio como el $75 \%$ de las víctimas del online grooming presentan edades entre los trece y diecisiete años. Por otro lado, Villacampa y Gómez (2016) sitúan la mayor probabilidad de convertirse en víctima de este delito a los menores entre quince y diecisiete años. "La persona que tiene menos posibilidades de ser víctima de 
groming, especialmente entre iguales, es el joven de catorce años" (p. 13).

En cuanto al sexo de las víctimas, mayoritariamente, son niñas. Villacampa y Gómez (2016) hallan en su investigación que el riesgo de convertirse en víctima de este delito se reduce siendo un niño. Asimismo, Peris y Maganto (como se citó en Maldonado, 2019) afirman que a pesar de que los niños cometen conductas más arriesgadas, siguen siendo las niñas las más victimizadas. "Esto podría explicar que (...), son las chicas las más victimizadas, quizás porque las estrategias más sutiles y elaboradas que emplea el agresor con ellas no les permita detectar y alertar las verdaderas intenciones enmascaradas" (Maldonado, 2019, p.9).

Por último, Webster et al. (2012) realizan una tipología de víctimas del online grooming. Esta categorización muestra que existen dos tipos: (i) aquellas extrovertidas y seguras de sí mismas, es decir, víctimas arriesgadas. "Estos sujetos se caracterizan por presentar una actitud desinhibida y arriesgada online" (Montiel et al., 2014, p.10); (ii) aquellos que presentan sentimientos de soledad y baja autoestima, con ambientes familiares conflictivos, es decir, víctimas vulnerables. "Aquellas con elevada necesidad de atención y afecto, con dificultades en la relación con los padres" (Montiel et al., 2014, p.10).

El objetivo principal del presente estudio es explorar, tal y como sugiere la literatura científica del online grooming, el perfil del potencial delincuente que perpetra este delito y sus diferentes tipologías. Esto puede proporcionar contenido que ayude en la prevención del impacto de este tipo concreto de delito y presente información que pueda ser utilizada en futuras investigaciones para facilitar el esclarecimiento del online grooming. Ahora bien, para alcanzar el objetivo principal, se establecen los objetivos secundarios siguientes:

1. Obtener los datos del perfil de groomer existente de dos regiones diferenciadas.

2. Estudiar los aspectos comunes y elaborar un perfil general.

3. Extraer las distintas tipologías de groomers del perfil común y contrastarlas con las de la literatura científica.

Las hipótesis, formuladas a raíz de la información extraída de la revisión de la literatura especializada, son las siguientes:

Hipótesis 1. La mayor concentración de groomers se encuentra en hombres jóvenes, por lo que a medida que la edad es mayor, menor será el número de individuos que perpetra este delito.

Hipótesis 2. Respecto a la prolongación del online grooming en el tiempo, se hipotetiza que el efecto de la prolongación del tiempo en la conversación entre agresor y víctima hace que la tipología hipersexualizada disminuya, permitiendo un aumento de los íntimos y adaptativos.

Hipótesis 3. Las estrategias empleadas por los groomers son más elaboradas y sutiles, por lo que será mayor el número de agresores que presenta una estrategia más desarrollada para ganarse la confianza del menor.

Hipótesis 4. El número de individuos que comete online grooming, prefiere un contacto sexual físico, a pesar de que el contacto y la proposición se realicen mediante las TICs.

\section{MÉtodo}

\section{A. Muestra}

La muestra la componen 258 casos, de los cuales 243 son casos de online grooming en Estados Unidos (EE. UU.) publicados desde el año 2003 hasta 2016, extraídos de una base de datos abierta ofrecida por Perverted Justice Foundation Incorporated (PJFI), una fundación sin ánimo de lucro, cuya finalidad principal reside en la lucha contra los depredadores sexuales online, y 15 son casos de online grooming en España publicados desde el año 2016 hasta 2018, extraídos del centro de documentación judicial (CENDOJ) del Consejo General del Poder Judicial (CGPJ).

Respecto a la muestra de EE. UU., se calculó a través del universo que ofrece PJFI de $\mathrm{N}=623$ conversaciones, con la finalidad de que el análisis tuviera aproximadamente un margen de error de entre un $3-5 \%$. Para obtener este cálculo se aplicó la ecuación I:

$$
n=\frac{N \times Z^{2} x p x q}{d^{2} x(N-1)+Z^{2} x p \times q}
$$

Para la ecuación I se utilizaron los siguientes parámetros: universo $(\mathrm{N}=623)$, nivel de confianza del $95 \%(\mathrm{Z}=1,96)$, precisión $(\mathrm{d}=0,03 \mathrm{y} \mathrm{d}=0,05)$ y proporción esperada $(\mathrm{p}=\mathrm{q}=0,5)$.

Con un error de un $3 \%$ se necesitarían aproximadamente una $n=394$ conversaciones y con un $5 \%$ de error la $n=238$. Por razones de tiempo, debido a la traducción y elaboración del análisis, la muestra es $n=243$. Por ello, se aplicó la ecuación II:

$$
\text { Error }_{\text {muestra }}=Z x \frac{\sigma}{\sqrt{n}}
$$

Para la ecuación II se empleó los siguientes 
parámetros: desviación estándar de la muestra $(\sigma=0,39)$, $\mathrm{n}=243$ conversaciones y un nivel de confianza del $95 \%$, donde el valor de $Z=1,96$. El resultado obtenido señala que la muestra seleccionada de $n=243$ presenta un margen de error de un $4,9 \%$.

Finalmente, se seleccionó mediante un método aleatorio simple una muestra del universo que ofrece PJFI de $\mathrm{N}=623$ conversaciones. Para ello, en primer lugar, se enumeró el universo ofrecido por PJFI del 1 al 623; en segundo lugar, se eligió al azar 243 conversaciones, ya que así todas las conversaciones tendrían las mismas posibilidades de ser seleccionadas; finalmente, esos 243 números elegidos, se corresponden con las conversaciones que conforman la muestra.

Respecto a la muestra española, se calculó a través del universo que ofrece el CENDOJ del CGPJ. La jurisprudencia española no emplea un único término para referenciar el fenómeno online grooming, por tanto, para el desarrollo de la muestra se utilizaron los términos child grooming, ciberacoso o ciberacoso sexual. El resultado ofreció un universo de $\mathrm{N}=20$ sentencias.

A continuación, se seleccionó a través de un método aleatorio simple una muestra del universo que ofrece el CENDOJ del CGPJ de $\mathrm{N}=20$ sentencias. Para ello, en primer lugar, se enumeró el universo ofrecido por el CENDOJ del CGPJ del 1 al 20; en segundo lugar, se eligió al azar 15 conversaciones, ya que así todas las sentencias tendrían las mismas posibilidades de ser seleccionadas; en tercer lugar, esos 15 números elegidos, se corresponderían con las sentencias que conforman la muestra, sin embargo, se observó que 2 de ellas no ofrecían todos los datos necesarios para el análisis, por tanto, se volvió a enumerar del 1 al 5 las sentencias que no resultaron elegidas y se seleccionaron 2 al azar; finalmente, se añadieron estas dos nuevas sentencias a las 13 seleccionadas de forma aleatoria en el paso anterior y, así, se obtuvo las sentencias que conforman la muestra. Por tanto, la muestra de sentencias utilizada es $n=15$. Asimismo, aplicando la ecuación II con una desviación estándar de la muestra $(\sigma=0,09), \mathrm{n}=15$ sentencias y un nivel de confianza del $95 \%$, donde el valor de $Z=1,96$, el resultado obtenido señala que la muestra presenta un margen de error de un 4,5\%.

\section{B. Procedimiento}

Para el presente estudio se emplea un método comparativo entre las dos fuentes de análisis. Esto permite contrastar la tipología ofrecida por los datos de EE. UU., con la ofrecida por los datos de España y, así, obtener un perfil común con el que comprobar, si es posible hallar diferentes tipologías de agresores dentro del fenómeno online grooming, alcanzando de esta forma los objetivos secundarios que permiten conseguir el objetivo principal.

Las variables utilizadas que nos permiten extraer el perfil de groomer existente son el sexo y la edad tanto de los agresores como de las víctimas, el tiempo entre el contacto y la proposición sexual a los menores, las estrategias empleadas para ganar la confianza de la víctima y el contacto sexual entre ambos sujetos. Asimismo, en caso afirmativo de producirse dicho encuentro, también se analiza el medio por el que se realiza el contacto sexual.

Para la codificación de la variable estrategias empleadas se agrupó en ocho grupos, en función de la característica principal de la técnica utilizada por el individuo que perpetraba el delito. Esto permitió obtener las siguientes categorías:

1. Estrategia Cero. La característica primordial reside en el hecho de que estos sujetos, no utilizaban ninguna técnica para ganarse la confianza del menor, es decir, manifestaban de forma directa su deseo de contacto sexual con la víctima.

2. Estrategia Uno. La principal característica de la técnica empleada por este grupo estaba en aprovechar el desconocimiento de la víctima sobre las relaciones sexuales. Así, despersonalizaban a la víctima, denominándolas «alumnos», ya que ellos serían los «maestros» que les introducirían en los «juegos de adultos».

3. Estrategia Dos. La característica de la técnica utilizada por estos agresores era la cautela empleada a la hora de seleccionar cuidadosamente las palabras para comunicarse con la víctima, creando así un falso ambiente «amistoso», donde el menor se encuentre cómodo y poco a poco ir ganándose su confianza.

4. Estrategia Tres. La característica de la técnica empleada por este grupo se encuentra en la protección que ofrecían a sus víctimas, puesto que éstas presentaban necesidad de atención y protección, la cual era ofrecida por el groomer. Así, las víctimas se mostraban más seguras.

5. Estrategia Cuatro. La característica primordial se encuentra en la intención de entablar una relación de amistad con el menor, para ello se adapta a su víctima mediante el humor, la música, la pasión por los animales, o bien, por el gusto de las artes culinarias. Finalmente, estas amistades generan una confianza que conduce al 
contacto sexual.

6. Estrategia Cinco. La técnica utilizada por estos groomers es establecer relaciones de índole romántica con los menores. Así, bajo la influencia de un supuesto romance obtendrían la confianza de su víctima.

7. Estrategia Seis. La característica principal de esta técnica es la adulación, es decir, mediante alabanzas interesadas generan confianza en el menor para obtener el contacto sexual.

8. Estrategia Siete. La característica que engloba la última categoría de estrategias, son aquellos groomers que utilizan técnicas agresivas y de dominación de la víctima, para obtener su objetivo primordial, que es el contacto sexual.

\section{Resultados}

De los 258 casos de la muestra comparada, todos fueron perpetrados por hombres. No obstante, el sexo de las víctimas muestra una mayor preferencia por las niñas menores de edad, ya que los niños representan un $8,1 \%$ del total de casos (Tabla 1).

Tabla 1.

GROOMERS (G) Y VICTIMAS (V) DE EE. UU. Y ESPAÑA, SEGÚN SU SEXO (EN\%).

\begin{tabular}{ccccccc}
\hline \hline & \multicolumn{2}{c}{ EE. UU. } & \multicolumn{2}{c}{ ESPAÑA } & \multicolumn{2}{c}{ TOTAL } \\
\hline Sexo & G & V & G & V & G & V \\
\hline \hline Hombres & 100,0 & 8,4 & 100,0 & - & 100,0 & 8,1 \\
\hline Mujeres & - & 91,6 & - & 100,0 & - & 91,9 \\
\hline \hline Total & \multicolumn{2}{c}{$(243)$} & \multicolumn{2}{c}{$(15)$} & \multicolumn{2}{c}{$(258)$} \\
\hline \hline
\end{tabular}

$\overline{\text { Fuente: Elaboración propia de acuerdo con la información extraída de }}$ las conversaciones de PJFI y sentencias del CENDOJ del CGPJ.

El rango de edad de los groomers es muy amplio, puesto que abarca desde los dieciocho hasta los sesenta y cinco años de edad. No obstante, los resultados extraídos de la muestra reflejan que el grupo mayoritario de agresores se sitúa con un $46,5 \%$ (Tabla 2 ) en la franja de edad entre los veintiuno y los treinta años.

La edad de las víctimas se sitúa en la franja que abarca desde los once hasta los quince años (Tabla 3), sin embargo, la franja de edad preferida de los agresores, se sitúa en menores entre los trece y catorce años, sólo estas dos edades, en conjunto, agrupan el $84,5 \%$ de las víctimas de la muestra.

Tabla 2.

GROOMERS (G) DE EE. UU. Y ESPAÑA, SEGÚN SU EDAD (EN\%).

\begin{tabular}{cccc}
\hline \hline & EE. UU. & España & Total \\
\hline Tramo de & $\mathrm{G}$ & $\mathrm{G}$ & $\mathrm{G}$ \\
\hline \hline
\end{tabular}

\begin{tabular}{cccc}
\hline edad & & & \\
\hline \hline Entre 18 y 20 & 2,4 & 13,3 & 3,1 \\
\hline Entre 21 y 30 & 47,8 & 26,7 & 46,5 \\
\hline Entre 31 y 40 & 24,7 & 40,0 & 25,6 \\
\hline Entre 41 y 50 & 14,8 & 13,3 & 14,7 \\
\hline Entre 51 y 65 & 10,3 & 6,7 & 10,1 \\
\hline \hline Total & $(243)$ & $(15)$ & $(258)$ \\
\hline \hline
\end{tabular}

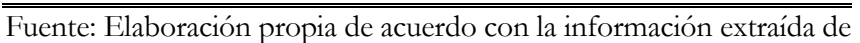
las conversaciones de PJFI y sentencias del CENDOJ del CGPJ.

Tabla 3.

VÍCTIMAS (V) DE EE. UU. Y ESPAÑA, SEGÚN SU EDAD (EN $\%)$.

\begin{tabular}{cccc}
\hline \hline & EE. UU. & España & Total \\
\hline Edad & $\mathrm{V}$ & $\mathrm{V}$ & $\mathrm{V}$ \\
\hline \hline 11 años & - & 13,3 & 0,8 \\
\hline 12 años & 9,5 & 53,4 & 12,0 \\
\hline 13 años & 53,1 & 13,3 & 50,8 \\
\hline 14 años & 35,3 & 6,7 & 33,7 \\
\hline 15 años & 2,1 & 13,3 & 2,7 \\
\hline \hline Total & $(243)$ & $(15)$ & $(258)$ \\
\hline \hline
\end{tabular}

Fuente: Elaboración propia de acuerdo con la información extraída de las conversaciones de PJFI y sentencias del CENDOJ del CGPJ.

Los resultados del tiempo que tarda el groomer desde que contacta con el menor hasta que realiza la proposición sexual al mismo de la muestra reflejan como el 50,8\% (Tabla 4) de los agresores tardan tan solo minutos desde el contacto hasta la proposición sexual. No obstante, los resultados que se aproximan a la realidad criminológica del online grooming son aquellos groomers que tardan un tiempo superior a horas, es decir, los que representan, en conjunto, un $29,4 \%$ de la muestra total.

Tabla 4.

GROOMERS (G) DE EE. UU. Y ESPAÑA, SEGÚN EL TIEMPO ENTRE EL CONTACTO Y LA PROPOSICIÓN SEXUAL (EN\%).

\begin{tabular}{cccc}
\hline \hline & EE. UU. & España & Total \\
\hline Tiempo & $\mathrm{G}$ & $\mathrm{G}$ & $\mathrm{G}$ \\
\hline \hline Segundos & 0,4 & - & 0,4 \\
\hline Minutos & 53,9 & - & 50,8 \\
\hline Horas & 20,6 & - & 19,4 \\
\hline Días & 23,5 & 26,7 & 23,6 \\
\hline Meses & 1,6 & 66,7 & 5,4 \\
\hline Años & - & 6,6 & 0,4 \\
\hline \hline Total & $(243)$ & $(15)$ & $(258)$ \\
\hline \hline
\end{tabular}

Fuente: Elaboración propia de acuerdo con la información extraída de las conversaciones de PJFI y sentencias del CENDOJ del CGPJ.

Los resultados de las diferentes estrategias utilizadas por los groomers para ganarse la confianza del menor y prepararlo para el contacto sexual, reflejan como el 81,5\% del total de la muestra (Tabla 5), lo engloban tres categorías: la estrategia cero, la cuatro y la seis. Los datos criminológicos que se pueden extraer para la configuración del perfil es que los groomers prefieren 
utilizar de menor a mayor proporción: la estrategia cuatro (14,8\% del total), es decir, entablar relaciones de amistad; la estrategia seis $(25,5 \%$ del total), es decir, la adulación y, finalmente, la estrategia cero ( $41,2 \%$ del total), es decir, manifestar directamente su intención de contacto sexual con los menores.

Tabla 5.

GROOMERS (G) DE EE. UU. Y ESPAÑA, SEGÚN LA ESTRATEGLA UTILIZADA (EN \%).

\begin{tabular}{cccc}
\hline \hline & EE. UU. & España & Total \\
\hline $\begin{array}{c}\text { Estrategia } \\
\text { utilizada }\end{array}$ & $\mathrm{G}$ & $\mathrm{G}$ & $\mathrm{G}$ \\
\hline \hline Cero & 41,2 & - & 41,2 \\
\hline Uno & 4,9 & - & 4,9 \\
\hline Dos & 6,2 & - & 6,2 \\
\hline Tres & 2,05 & - & 2,05 \\
\hline Cuatro & 14,8 & - & 14,8 \\
\hline Cinco & 3,3 & - & 3,3 \\
\hline Seis & 25,5 & - & 25,5 \\
\hline Siete & 2,05 & - & 2,05 \\
\hline \hline Total & $(243)$ & $(0)$ & $(243)$ \\
\hline \hline Casos sin & 0 & 15 & 15 \\
datos & & & \\
\hline Fut & & &
\end{tabular}

Fuente: Elaboración propia de acuerdo con la información extraída de las conversaciones de PJFI y sentencias del CENDOJ del CGPJ.

Tabla 6.

GROOMERS (G) DE EE. UU. Y ESPAÑA, SEGÚN LA ESTRATEGLA UTILIZADA CON UN TIEMPO, ENTRE CONTACTO Y PROPOSICIÓN SEXUAL, SUPERIOR A HORAS (EN\%).

\begin{tabular}{cccc}
\hline \hline & EE. UU. & España & Total \\
\hline $\begin{array}{c}\text { Estrategia } \\
\text { utilizada }\end{array}$ & $\mathrm{G}$ & $\mathrm{G}$ & $\mathrm{G}$ \\
\hline \hline Cero & 4,9 & - & 4,9 \\
\hline Uno & 1,6 & - & 1,6 \\
\hline Dos & 6,6 & - & 6,6 \\
\hline Tres & 3,3 & - & 3,3 \\
\hline Cuatro & 23,0 & - & 23,0 \\
\hline Cinco & 4,9 & - & 4,9 \\
\hline Seis & 55,7 & - & 55,7 \\
\hline Siete & - & - & - \\
\hline \hline Total & $(61)$ & $(0)$ & $(61)$ \\
\hline \hline Casos sin & 0 & 15 & 15 \\
datos & & & \\
\hline \hline
\end{tabular}

Fuente: Elaboración propia de acuerdo con la información extraída de las conversaciones de PJFI y sentencias del CENDOJ del CGPJ.

Para ahondar en las distintas tipologías de groomers existentes, el presente estudio analiza las estrategias utilizadas por los groomers, pero solo en aquellos individuos que presentan un tiempo entre el contacto y la proposición sexual superior a la hora. Los resultados de la muestra reflejan que la obtención de confianza de la víctima y su preparación para el contacto sexual, requiere tiempo y estrategias elaboradas. Por ello, se observa
(Tabla 6) una reducción de la estrategia cero a un 4,9\%, ya que ésta se caracteriza por no ganarse la confianza de la víctima, sino manifestar de manera directa su intención sexual. Asimismo, las estrategias cuatro y seis aumentan, obteniendo un $23 \%$ y un $55,7 \%$ respectivamente.

Los resultados muestran como en un 1,6\% de los 258 casos, el contacto sexual no llega a producirse, por lo que en un $98,4 \%$ del total de la muestra sí se produce contacto sexual entre el groomer y la víctima, a pesar de producirse el contacto y la proposición sexual mediante TICs (Tabla 7). Asimismo, en el 88,6\% del total de casos en los que sí se produce el contacto, los groomers prefieren el medio físico con los menores, frente al $11,4 \%$ de los individuos que perpetran este fenómeno a través de las TICs (Tabla 8).

Tabla 7.

GROOMERS (G) DE EE. UU. Y ESPAÑA, SEGÚN LA PRESENCLA DE CONTACTO SEXUAL (EN \%).

\begin{tabular}{cccc}
\hline \hline & EE. UU. & España & Total \\
\hline $\begin{array}{c}\text { Contacto } \\
\text { sexual }\end{array}$ & $\mathrm{G}$ & $\mathrm{G}$ & $\mathrm{G}$ \\
\hline \hline Sí & 98,4 & 100,0 & 98,4 \\
\hline No & 1,6 & - & 1,6 \\
\hline \hline Total & $(243)$ & $(15)$ & $(258)$ \\
\hline \hline
\end{tabular}

Fuente: Elaboración propia de acuerdo con la información extraída de las conversaciones de PJFI y sentencias del CENDOJ del CGPJ.

Tabla 8.

GROOMERS (G) DE EE. UU. Y ESPAÑA, SEGÚN EL MEDIO DE CONTACTO SEXUAL (EN \%).

\begin{tabular}{cccc}
\hline \hline & EE. UU. & España & Total \\
\hline Medio & $\mathrm{G}$ & $\mathrm{G}$ & $\mathrm{G}$ \\
\hline \hline Físico & 89,1 & 80,0 & 88,6 \\
\hline TICs & 10,9 & 20,0 & 11,4 \\
\hline \hline Total & $(239)$ & $(15)$ & $(254)$ \\
\hline \hline
\end{tabular}

$\overline{\text { Fuente: Elaboración propia de acuerdo con la información extraída de }}$ las conversaciones de PJFI y sentencias del CENDOJ del CGPJ.

\section{Discusión}

Este perfil de los individuos que perpetran online grooming se aproxima al extraído de la revisión de la literatura especializada, que permitía establecer cuatro hipótesis que vertebraban el análisis.

La literatura especializada indicaba que este fenómeno se perpetraba predominantemente por hombres que no tenían más de veinticinco años de edad (Villacampa y Gómez, 2016 y De Santisteban y Gámez Gaudix, 2017b), aunque estudios recientes apuntaban una aparición de las mujeres groomers. En primer lugar, los datos de la muestra conjunta indican una predominancia masculina, ya que la población total de groomers es desconocida, por lo que no podemos demostrar que no haya ninguna mujer groomer. En segundo lugar, según la muestra comparada de este 
estudio señala como el rango de edad mayoritario, lo componen individuos entre los veintiuno y los treinta años. Esto demuestra que el online grooming es un delito joven, ya que los perpetradores se sitúan en un rango de edad muy cercano al que indica la literatura científica. Por tanto, la primera hipótesis se cumple, puesto que a medida que aumenta la edad, el número de groomers se reduce.

La literatura especializada mostraba la existencia de múltiples categorizaciones dependiendo del criterio seleccionado. Webster et al. (2012) identificaron tres tipos, según la motivación principal del autor, es decir, los groomers íntimos, adaptativos e hipersexualizados. La muestra conjunta, refleja la existencia de tres tipos de groomers, cuya motivación principal puede ser sexual, o bien, afectiva. La sexual engloba a los adaptativos y los hipersexualizados. La diferencia entre un tipo u otro, dependía de la capacidad del sujeto de obtener un encuentro sexual inmediato, utilizando una estrategia cero, es decir, aquellos sujetos que manifestaban de forma directa su deseo de contacto sexual con la víctima y un tiempo inferior a horas, o bien adaptarse a las necesidades de la víctima, mediante el empleo de una estrategia seis, o sea, mediante alabanzas interesadas generan confianza en el menor para obtener el contacto sexual y un tiempo superior a horas. En cuanto a la motivación afectiva, la estrategia utilizada era la número cuatro, aquella cuya intención es entablar una relación de amistad real con el menor y el tiempo era superior a horas. Por tanto, la muestra comparativa de esta investigación refleja la existencia de la tipología señalada por la literatura científica. Asimismo, la tercera hipótesis se cumple, puesto que a medida que se prolonga el tiempo entre el contacto y la proposición, disminuye el número de groomers hipersexualizados, aumentando la cantidad de individuos adaptativos e íntimos, e incluso se aprecia lo que se puede denominar un periodo de incertidumbre entre el tipo íntimo y el adaptativo, con un aumento de los groomers que utilizan una estrategia dos, o sea, seleccionar cautelosamente las palabras para comunicarse con la víctima, creando así un falso ambiente «amistoso».

La tercera hipótesis establecía que las estrategias empleadas por los groomers son más elaboradas y sutiles, por lo que será mayor el número de agresores que presenta una estrategia más desarrollada para ganarse la confianza del menor (Maldonado, 2019). La literatura especializada señalaba que al ser las niñas las víctimas más vulnerables, las técnicas empleadas deberían ser más desarrolladas e ingeniosas, sin embargo, la muestra comparativa de este análisis indica lo contrario, pues la estrategia más utilizada es aquella que manifiesta de forma directa su deseo de contacto sexual con la víctima. Por tanto, la cuarta hipótesis no se cumple, esto puede deberse a que las sociedades se encuentran cada vez más sexualizadas y las relaciones de índole sexual se alejan del mito romántico y el proceso de cortejar a la otra persona, aumentando así los encuentros sexuales inmediatos, por ejemplo, en los últimos tiempos se observa un incremento de las redes sociales para ligar, tales como Tinder o Grindr, mediante las cuales se producen numerosos encuentros sexuales.

La última hipótesis que vertebraba este análisis indicaba que el número de individuos que comete online grooming, prefiere un contacto sexual físico (De Santisteban y Gámez-Gaudix, 2017b), a pesar de que el contacto y la proposición se realicen mediante las TICs. La muestra conjunta del análisis de esta investigación, representa que la mayoría de groomers analizados en este estudio mantienen un contacto sexual con los menores y, dentro de este amplio grupo, los individuos que cometen online grooming optan, mayormente, por un contacto sexual físico, aunque el contacto y la propuesta se haya realizado mediante la red. Por tanto, la cuarta hipótesis también se cumple, ya que la preferencia de los groomers estudiados en esta investigación consiste en mantener un contacto sexual físico, aunque el contacto y proposición previa se produzca de forma online.

Con todo lo mencionado anteriormente, se observa como este perfil común es imperfecto, tampoco es universal, ya que como sucede en todos los campos delictivos existe la cifra negra u oscura y, por ello, no disponemos de todos los datos reales, sin embargo, al ser un análisis, que utiliza datos oficiales, aunque existan numerosas críticas, este perfil extraído confirma la existencia del fenómeno online grooming en dos países muy diferentes, concuerda con la información que nos ofrece la literatura especializada en este delito y refleja la existencia de diferentes tipologías de groomers.

\section{CONCLUSIONES Y LIMITACIONES}

La visión criminológica del delito online grooming que ofrece el presente estudio, destaca las estrategias empleadas por el individuo que perpetra este delito como un elemento fundamental del desarrollo que se produce entre agresor y víctima con la finalidad de obtener la confianza de ésta última. Las técnicas más destacadas de la literatura científica son aquellas que persiguen la muestra de afecto o cariño, el engaño, la manipulación, o bien, la agresividad o violencia. 
Los resultados del análisis han permitido una aproximación al perfil de los groomers que perpetran este delito, sin embargo, la elaboración de éste presenta una serie de limitaciones:

En primer lugar, al tratarse de un delito sexual cometido a través de las TICs, las cifras oficiales no abarcan todos los casos reales, ya que las víctimas pueden no percibirse como tales, o bien porque los menores ignoran que se encuentran en un delito castigado penalmente.

En segundo lugar, se seleccionaron las sentencias españolas que respondían a los términos jurídicos child grooming, ciberacoso o ciberacoso sexual para denominar al delito online grooming, sin embargo, cabe la posibilidad de que exista una mayor muestra de este fenómeno en nuestro país, que no utilice esos términos y no haya aparecido en la búsqueda.

En tercer lugar, el lenguaje escrito inglés caracterizado por el uso de múltiples abreviaturas, la utilización de letras o números para sustituir palabras, por ejemplo, «idk» por «I don't knou»» «U» por «you» o «2nite» por «tonight», dificulta la traducción, ya que puede perderse matices en las estrategias utilizadas por los groomers.

Finalmente, la última limitación la encontramos en las estrategias utilizadas por los agresores. En esa variable se realizó una agrupación, según la característica principal de la técnica empleada, sin embargo, otro investigador podría seleccionar otro criterio completamente válido dentro del online grooming y obtener otro resultado distinto al que muestra el análisis. Asimismo, en el caso de España, la información extraída de los hechos probados, no representa que aquellos datos que no pudieron demostrarse a lo largo del procedimiento judicial, no se hubieran realizado.

El perfil extraído, teniendo en cuenta las limitaciones, representa al groomer como un hombre de entre veintiuno y treinta años de edad, motivado, con carácter general, por niñas adolescentes de trece años. Esta motivación puede ser sexual, o bien, afectiva. Cuando el groomer presenta una motivación sexual, ésta puede clasificarse en dos tipos: inmediata o tardía. Esto refleja los tipos diferenciados de groomers que se encuentran en este fenómeno: íntimos, adaptativos e hipersexualizados.

La prolongación del tiempo entre el contacto y la proposición, nos muestra las capacidades del groomer para ganarse la confianza y preparar al menor para el contacto sexual. Cuando la prolongación del tiempo es superior a horas, las estrategias utilizadas por los individuos requieren un cierto grado de elaboración y sofisticación, por lo que las técnicas más empleadas fueron: por un lado, mediante alabanzas interesadas generando, así, confianza en el menor para obtener el contacto sexual y, por otro lado, entablar una relación de amistad real con el menor, de tal manera que la presencia de la tipología hipersexualizada se reduce, aumentado la de los íntimos y adaptativos.

Por tanto, esta investigación muestra datos significativos que pueden servir como fundamento para plantear nuevas hipótesis criminológicas y analizarlas con mayor determinación, así como con una profundidad más extensa, a pesar de que pueda ser un análisis muy criticado, también podría ser utilizado para el desarrollo de software, por ejemplo, crear medios TICs como redes sociales o chats online que detecten en el intercambio de mensajes entre perfiles que interactúan y bloqueé la mensajería entre ambos sujetos cuando detecte alguna anomalía en la conversación, o bien, que no permita a perfiles de adultos interaccionar con menores desconocidos. Asimismo, este contenido puede utilizarse para elaborar guías prácticas, desde una perspectiva criminológica, que generen, por un lado, seguridad a los padres ofreciéndoles ayuda en la prevención del impacto que el online grooming pueda provocar en los menores y, por otro lado, pueden servir a los menores, enseñándoles cómo utilizar correctamente las TICs. Además, las autoridades pueden emplear estos datos en la detección de perfiles en las redes sociales que cometan este delito y proporcionar información que facilite el esclarecimiento del online grooming, no obstante, se requiere futuras investigaciones que profundicen en este fenómeno.

\section{REFERENCIAS}

Bergen, E., Ahto, A., Schulz, A., Imhoff, R., Antfolk, J., Schuhmann, P., Alanko, K., Santtila, P., \& Jern, P. (2014). Adult-Adult and Adult-Child/Adolescent Online Sexual Interactions: An Exploratory Self-Report Study on the Role of Situational Factors. The Journal of Sex Research, 52, 1006 1016. https://doi.org/10.1080/00224499.2014.914462.

Briggs, P., Simon, W. T., \& Simonsen, S. (2011). An exploratory study of Internet-initiated sexual offenses and the chat room sex offender: Has the Internet enabled a new typology of sex offender? Sexual Abuse, 23, 72 - 91. https://doi.org/10.1177/1079063210384275.

Craven, S., Brown, S., \& Gilchrist, E. (2006). Sexual grooming of children: Review of literature and theoretical considerations. Journal of Sexual Aggression, 12 (3), 287 - 299.

De Santisteban, P. y Gámez-Gaudix, M. (2017a). Online 
grooming y explotación sexual de menores a través de internet. Revista de Victimología, 6, 81 - 100.

De Santisteban, P. y Gámez-Guadix, M. (2017b). Prevalence and risk factors among minors for online sexual solicitations and interactions with adults. Journal of Sex Research, 1 - 12.

Gámez-Guadix, M., De Santisteban, P. y Alcázar, M. Á. (2017). The construction and psychometric properties of the questionnaire for online sexual solicitation and interaction of minors with adults. Sexual Abuse: A Journal of Research and $\begin{array}{lllll}\text { Treatment, } & 6, & 81 & - & 100 .\end{array}$ https://doi.org/10.1177/1079063217724766.

Kloess, J. A., Seymour-Smith, S., Hamilton-Giachritsis, C. E., Long, M. L., Shipley, D., \& Beech, A. R. (2017). A qualitative analysis of offenders' modus operandi in sexually exploitative interactions with children online. Sexual Abuse: A Journal of Research and Treatment.

Maldonado Guzmán, D. J. (2019). El mal denominado delito de grooming online como forma de violencia sexual contra menores. Problemas jurídicos y aspectos criminológicos. Revista Electrónica de Estudios Penales y de la Seguridad, 5, 1 - 18.

Montiel Juan, I., Carbonell-Vayá, E. y Salom García, M. (2014). Victimización infantil sexual online: online grooming, ciberabuso y ciberacoso sexual. En Lameiras Fernández, M. y Orts Berenguer, E. Delitos sexuales contra menores abordaje psicológico, jurídico y policial (pp. 203 - 224). Valencia, España: Tirant lo Blanch.

O'Connell, R. (2003). A typology of cyber sexploitation and online grooming practices. Preston, England: University of Central Lancashire.

Seto, M. C., Wood, J. M., Babchishin, K. M., \& Flynn, S. (2012). Online solicitation offenders are different from child pornography offenders and lower risk contact sexual offenders. Law and Human Behavior, 36(04), 320 - 330. https://doi.org/10.1037/h0093925.

Tener, D., Wolak, J., \& Finkelhor, D. (2015). A typology of offenders who use online communications to commit sex crimes against minors. Journal of Aggression, Maltreatment y $\begin{array}{lllll}\text { Trauma, } & 24, & 319 & - & 339 .\end{array}$ https://doi.org/10.1080/10926771.2015.1009602.

Viader Castro, C. (12 de mayo de 2020). La delincuencia que no descansa en el coronavirus: child grooming y pornografía infantil [Mensaje en un blog]. Recuperado de http://www.derechopractico.es/la-delincuencia-que-nodescansa-en-el-coronavirus-child-grooming-y-pornografiainfantil/

Villacampa Estiarte, C. (2014). Propuesta sexual telemática a menores $\mathrm{u}$ online child grooming: configuración presente del delito y perspectivas de modificación. Estudios penales y criminológicos, 34, $639-712$.

Villacampa Estiarte, C. y Gómez Adillón, M. J. (2016). Nuevas tecnologías y victimización sexual de menores por online grooming. Revista electrónica de ciencia penal y criminología, 18(2). $1-27$.

Webster, S., Davidson, J., Bifulco, A., Gottschalk, P., Caretti, V., Pham, T., Grove-Hills, J., Turley, C., Tompkins, C., Ciulla, S. Milazzo, V., Schimmenti, A., \& Craparo, G. (2012). Final report. European Online Grooming Project. London: European Comission Safer Internet Plus Programme.

Young, K. (2005). Profiling online sex offenders, cyberpredators and pedophiles. Journal of Behavioral Profiling, 5(01), $1-18$. 